Case Report

\title{
Teratoma with Naturally Occurring Malignant Transformation in a Child
}

\author{
Mamoru Takahashi, MD, Norihito Okumura, MD, Tomoaki Matsuoka, MD,
}

Akihiko Yamashina, MD, Jun Nakano, MD, and Kotaro Kameyama, MD

\begin{abstract}
We present a 12-year-old girl with a teratoma with malignant transformation (TMT) of the mediastinum. Computed tomography showed a cystic mass $(5.0 \mathrm{~cm} \times 4.0 \mathrm{~cm})$ with a thick solid portion, in the anterior mediastinum. Six months later, the solid portion of the mass had enlarged, and surgical resection was performed. The resected tumor was $7.0 \times 5.0 \times 4.0 \mathrm{~cm}$ in size. The cystic portion was a mature teratoma, and the solid portion predominantly comprised a viable embryonal rhabdomyosarcoma. There were no immature teratomatous elements or other germ-cell components. The histopathologic diagnosis was a mature teratoma with embryonal rhabdomyosarcoma, a so-called TMT. The tumor recurred, despite adjuvant chemotherapy. The patient died of progressive disease 16 months postoperatively. To the best of our knowledge, no naturally occurring TMT of the mediastinum has previously been reported in a child. Surgical resection at an early stage is necessary.
\end{abstract}

Keywords: mediastinal teratoma, malignant transformation

\section{Introduction}

Teratomas are commonly found in the gonads and extragonadal sites, such as the mediastinum. Carcinomatous or sarcomatous components are occasionally found in pre-existing mature teratomas, and these are referred to as teratomas with malignant transformation (TMTs). ${ }^{1)}$ In the 2004 World Health Organization classification of tumors, mediastinal TMT is defined as a germ-cell tumor, accompanied by a somatic-type malignant component of sarcoma, carcinoma, or both, and is now classified as a 'germ-cell tumor with somatic-type malignancy. $^{2)}$

\footnotetext{
Department of Thoracic Surgery, Kurashiki Central Hospital, Kurashiki, Okayama, Japan
}

Received: January 5, 2011; Accepted: January 21, 2011

Corresponding author: Mamoru Takahashi, MD. Department of Thoracic Surgery, Kurashiki Central Hospital, 1-1-1 Miwa, Kurashiki, Okayama 710-8602, Japan

Email: mt10947@yahoo.co.jp

(C)2011 The Editorial Committee of Annals of Thoracic and Cardiovascular Surgery. All rights reserved.
In a review of the literature, Moran and Suster reported that mediastinal mature teratomas with rhabdomyosarcomas accounted for only four of 322 germ-cell tumors in the mediastinum. ${ }^{3)}$ However, the precise incidence of mediastinal TMT is not known. We present a rare case of mediastinal TMT and a literature review.

\section{Case Report}

The patient was a 12-year-old girl who presented with a mediastinal mass. The mass was accidentally found during a medical checkup. She was initially referred to the department of pediatrics of our hospital. Her history was unremarkable. A computed tomography (CT) scan revealed a lobulated cystic mass with a thick solid portion, in the anterior mediastinum.

The mass measured $5.0 \mathrm{~cm} \times 4.0 \mathrm{~cm}($ Fig. 1A). The wall of the solid portion was not well defined. There was no associated pleural effusion or mediastinal lymph node enlargement.

Tumor markers, including serum alpha-fetoprotein and human chorionic gonadotropin, were within normal limits. No distant metastases were detected by whole-body 


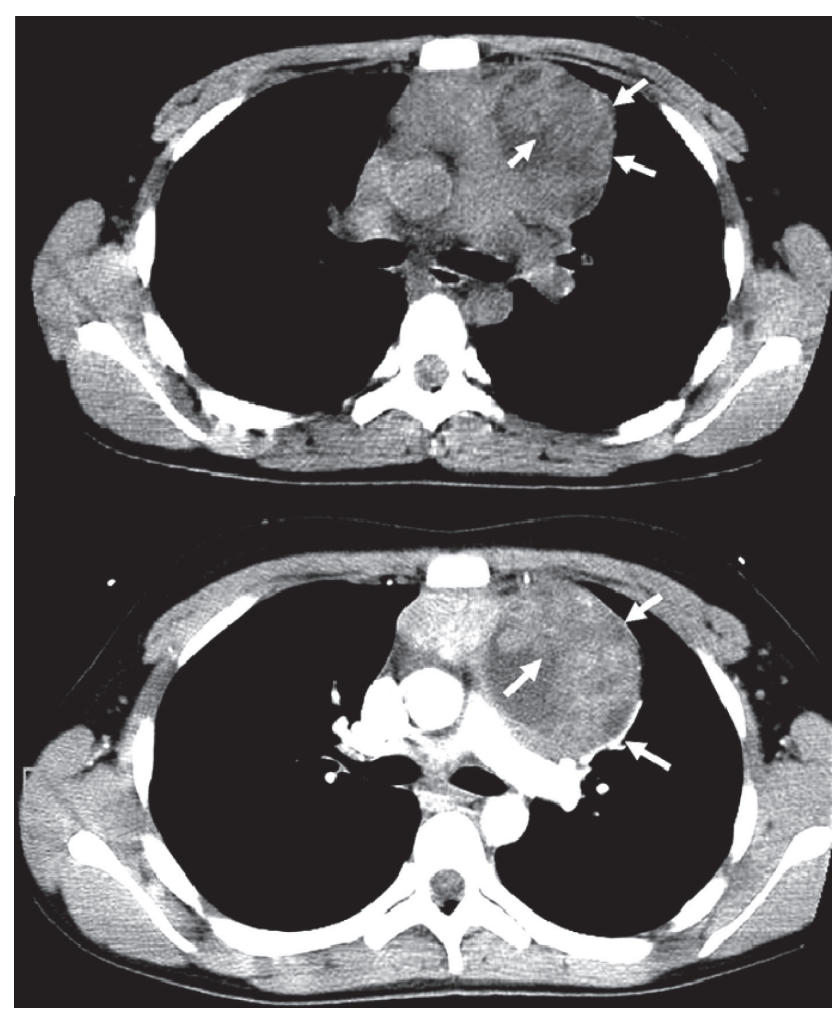

Fig. 1 (A) CT scan showing a lobulated mass with cystic portion and thick solid portion (white arrows) in the anterior mediastinum. (B) Six months later, the solid portion of the mass had clearly enlarged.

CT scan and magnetic resonance imaging. The clinical diagnosis of benign mature cystic teratoma was accepted. Although the possibility of malignancy could not be ruled out, no consensus on the surgical procedure, including biopsy, was obtained from the patient and her family, and radiographic follow-up was, therefore, continued every three months. At the 3-month follow-up, the mass did not show any changes.

At the 6-month follow-up, the solid portion of the mass had clearly enlarged (Fig. 1B); thus, she was referred to the thoracic surgery department. The patient and her family agreed to surgical treatment. Surgical resection was performed through a median sternotomy approach. The tumor was located in the anterior mediastinum and was found arising from the thymus, with no invasion of the left lung or great vessels in the mediastinum. The tumor was completely excised. Examination of frozen sections confirmed the diagnosis of a mature teratoma. The postoperative course was uneventful.

The resected tumor was $7.0 \times 5.0 \times 4.0 \mathrm{~cm}$ in size and weighed $135 \mathrm{~g}$ (Fig. 2A). The cystic portion was a mature

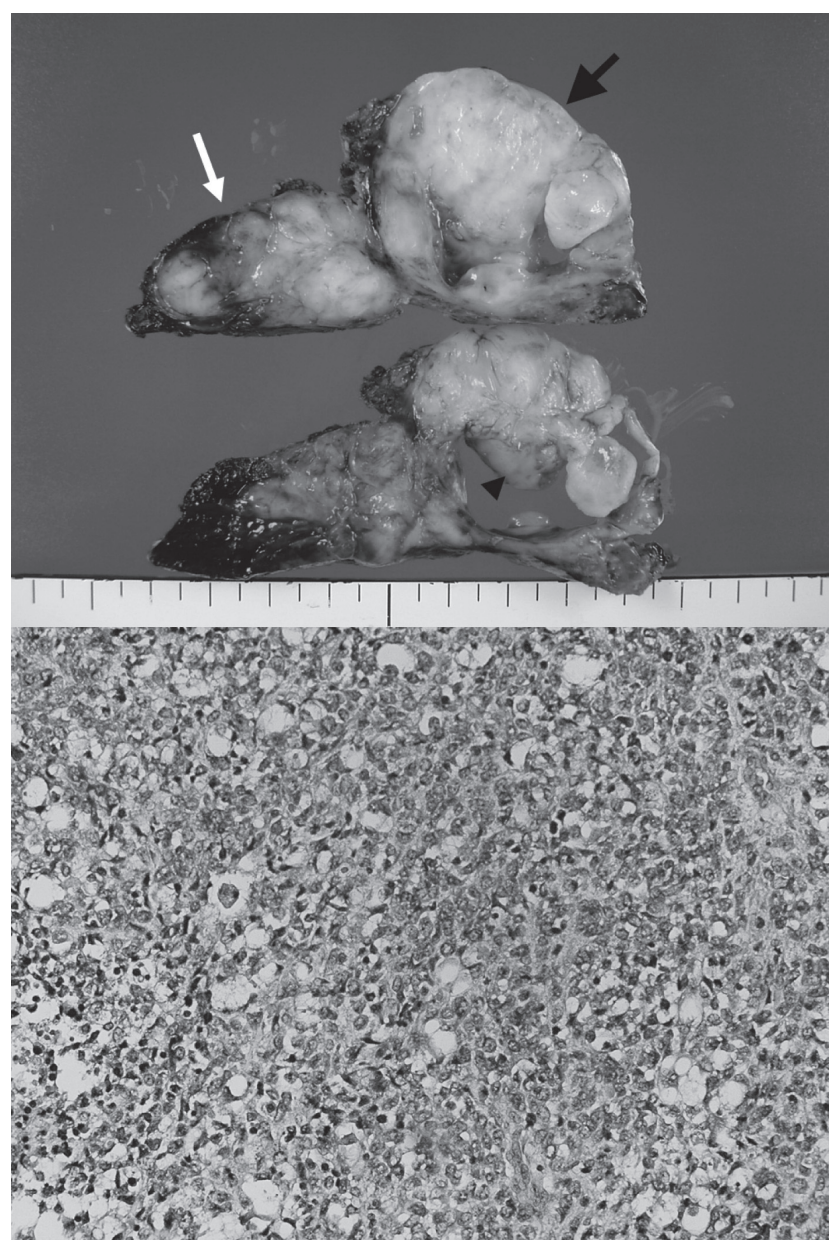

Fig. 2 (A) Gross appearance of the resected tumor. Mature teratoma was noted in the cystic portion (arrowhead) and a rhabdomyosarcoma occupied the solid portion of the tumor (black arrow). The tumor arose from the thymus (white arrow). (B) Microscopic findings of rhabdomyosarcoma in the resected tumor, showing sarcomatous component.Mitotic activity and prominent nucleoli were noted (hematoxylin and eosin, original magnification $\times 100$ ).

teratoma, while the solid portion predominantly comprised a viable embryonal rhabdomyosarcoma (Fig. 2B). There were no immature teratomatous elements or other germ-cell components. The histopathologic diagnosis was a mature teratoma with embryonal rhabdomyosarcoma, a so-called TMT. Adjuvant chemotherapy adapted to rhabdomyosarcoma (vincristine, actinomycin D, and cyclophosphamide) was initiated. No tumor was detectable by whole-body CT scan after eight cycles of treatment.

A CT scan at the 2-month follow-up revealed tumor recurrence and second-line chemotherapy was started. However, distant metastases were subsequently detected in the femur, the kidney and the liver, and the patient died 
of disease progression 16 months after operation. Autopsy revealed that all the relapsed tumors were embryonal rhabdomyosarcomas.

\section{Discussion and Conclusion}

TMTs have been classified into two clinical groups: TMTs induced by chemotherapy or irradiation, and naturally occurring TMTs. ${ }^{4)}$ The latter type has rarely been reported, ${ }^{5)}$ and cases in the literature are exclusively carcinomatous in older patients with long-standing, mature teratomas. ${ }^{4-6)}$ To the best of our knowledge, no naturally occurring TMT of the mediastinum has previously been reported in a child. This case suggests that malignant transformation can occur naturally in a child and that the tumor can grow over a short period.

Teratomas that are likely to undergo malignant transformation can be differentiated from others on the basis of radiographic features. Mediastinal tumors with invading solid portions or indistinct margins may be foci for malignant transformation. ${ }^{6}$ In the current case, the histopathologic report revealed a malignant focus in the solid portion of the tumor. But it was very difficult to detect when the somatic-type malignancy arose. The tumor might have already been a teratoma with rhabdomyosarcoma at the time of the first medical checkup.

TMT is usually very aggressive and fatal within a few months of the initial diagnosis, as a result of local spread and metastasis. Surgery plays an important role when the tumor is located at a single site. ${ }^{7}$ Complete surgical resection is the most important prognostic factor, and the postoperative prognosis is good if the tumor has not extended beyond the capsule. ${ }^{4}$

Chemotherapy should be adapted to the malignant components. However, the malignant components are generally refractory to chemotherapy, and embryonal rhabdomyosarcomas tend to be particularly unresponsive to chemotherapy regimens. The prognosis of mediastinal rhabdomyosarcomas is worse than that of their gonadal counterparts. ${ }^{8)}$ Early resection before disease progression is essential.

In conclusion, we present a case of a naturally occurring TMT in a child. TMT can occur in children and can grow over a short period. Malignant transformation should be suspected when a mediastinal teratoma with a thick solid portion and indistinct margin is detected. Surgical resection at an early stage is essential for a good prognosis.

\section{References}

1) Chen $C$, Zheng H, Jiang S. An unusual case of giant mediastinal teratoma with malignant transformation. Ann Thorac Surg 2008; 86: 302-4.

2) Wick MR, Perlman EJ, Strobel Ph, et al. Germ cell tumours with somatic-type malignancy. In: Travis WD, Brambilla E, Müller-Hermelink HK, Harris CC, editors. World Health Organization Classification of Tumours. Pathology and Genetics of Tumours of the Lung, Pleura, Thymus and Heart. Lyon: IARC Press; 2004: pp 216-8.

3) Moran CA, Suster S. Primary germ cell tumors of the mediastinum. Cancer 1997; 80: 681-90.

4) Morinaga S, Nomori H, Kobayashi R, et al. Well-differentiated adenocarcinoma arising from mature cystic teratoma of the mediastinum (teratoma with malignant transformation): report of a surgical case. Am J Clin Pathol 1994; 101: 531-4.

5) Shimizu K, Nakata M, Hirami Y, et al. Teratoma with malignant transformation in the anterior mediastinum. J Thorac Cardiovasc Surg 2008; 136: 225-7.

6) Jung JI, Park SH, Park JG, et al. Teratoma with malignant transformation in the anterior mediastinum: a case report. Korean J Radiol 2000; 1: 162-4.

7) Donadio AC, Motzer RJ, Bajorin DF, et al. Chemotherapy for teratoma with malignant transformation. J Clin Oncol 2003; 21: 4285-91.

8) Omezzine N, Khouatra C, Larive S, et al. Rhabdomyosarcoma arising in mediastinal teratoma in an adult: a case report. Ann Oncol 2002; 13: 323-6. 